\title{
ANÁLISE QUALITATIVA NO MONITORAMENTO AUTOMÁTICO DOS EVENTOS DE TENSÃO DE CURTA DURAÇÃO
}

\author{
Wesley F. Usida*
}

Mário Oleskovicz*

\author{
Letícia C. P. Rodrigues*
}

Denis V. Coury*

\author{
*SEL-EESC-USP São Carlos \\ Av. Trabalhador Sancarlense, 400 Centro \\ CEP 13566-590 - São Carlos - SP
}

\section{RESUMO}

Esta pesquisa busca caracterizar o monitoramento automático de eventos de curta duração relacionados à qualidade da energia elétrica (QEE). Toda a metodologia e resultados a serem apresentados tomarão como base um sistema de distribuição real, simulado e analisado via utilização do software ATP (Alternative Transients Program). Duas técnicas foram estudadas e comparadas no intuito de detectar e classificar os eventos de curta duração gerados. Frente às situações analisadas, os resultados obtidos demonstraram a eficiência da transformada Wavelet (TW) em comparação com o cálculo do valor RMS (Root Mean Square). Desta constatação, têmse pontos relevantes que abrem espaço para aplicações de novas técnicas de análise de sinais, como a TW, em QEE.

PALAVRAS-CHAVE: Qualidade da Energia Elétrica, Variação de Tensão de Curta Duração, Transformada Wavelet, valor RMS.

\section{ABSTRACT}

This research characterizes the automatic supervision of short-duration events concerning electrical power quality (PQ). The methodology and results are based on an actual

Artigo submetido em 18/08/2008 (Id.: 00886)

Revisado em 12/02/2009, 18/05/2009, 05/06/2009

Aceito sob recomendação do Editor Associado Prof. Julio Cesar Stacchini Souza distribution electric system that was simulated and analyzed using ATP (Alternative Transients Program) software. Two techniques were studied and compared to detect and classify the short-duration events. Concerning the situation studied, the results obtained have shown that the Wavelet transform (WT) is more efficient than calculation of RMS value. The evidences observed open space to applications of new techniques of analysis of the signal as WT in PQ.

KEYWORDS: Power Quality, Short-Duration Voltage Variation, Wavelet Transform, RMS value.

\section{INTRODUÇÃO}

Nos últimos anos houve grandes mudanças no setor elétrico brasileiro com a entrada de recursos privados. Tais mudanças provocaram uma profunda reestruturação nos serviços públicos de energia elétrica, já que vários destes passaram a ser concessões para diversos e distintos investidores.

Estas mudanças objetivaram assegurar o suprimento e também a qualidade no fornecimento da energia elétrica. Com isso, a busca por esta qualidade passou a ser preocupação de consumidores, agentes e empresas do setor elétrico nacional.

A Agência Nacional de Energia Elétrica (ANEEL), ciente da importância da qualidade da energia elétrica (QEE), publicou resoluções exigindo das empresas concessionárias o atendimento a certos indicadores de qualidade no forneci- 
mento. Dentre essas resoluções, destacam-se as de núme$\operatorname{ros}$ 024/2000 (ANEEL, 2000) e 505/2001 (ANEEL, 2001). A primeira trata da continuidade do fornecimento da energia elétrica, e a segunda, da conformidade de tensão em regime permanente.

Recentemente, a ANEEL, em conjunto com representantes do setor elétrico, elaborou um conjunto de diretrizes denominado "Procedimentos de Distribuição de Energia Elétrica no Sistema Elétrico Nacional - PRODIST", com a intenção de centralizar todos os aspectos de regulação a serem seguidos pelas empresas concessionárias e permissionárias de serviços de distribuição de energia elétrica (ANEEL, 2009).

Cabe colocar que o PRODIST está dividido em oito módulos, sendo o último direcionado aos aspectos relacionados à QEE. Tal módulo deixa claro duas frentes relacionadas à QEE: a qualidade do produto e a qualidade do serviço.

Dentre os diversos problemas relacionados à qualidade do produto, tem-se os relativos aos níveis de tensão que se refletem nas variações de tensão de curta duração (VTCDs) e variações de tensão de longa duração (VTLDs). Vale lembrar que afundamento, elevação e interrupção momentânea ou temporária de tensão integram os fenômenos referentes à classe das VTCDs. Sendo subtensão, sobretensão e interrupção sustentada alocados à classe das VTLDs. Além das magnitudes envolvidas e frequência $(60 \mathrm{~Hz})$ destas variações, quando comparadas à componente fundamental, o tempo de permanência destas sobre o sistema é o fator primordial para classificá-las entre VTCDs e VTLDs. No primeiro caso, o tempo associado é menor ou igual a três minutos, e em consequência deste, para o segundo caso, maior do que três minutos (ANEEL, 2009).

O presente trabalho tem como objetivo estudar os eventos de VTCDs, detectando e localizando no tempo, bem como classificando os mesmos dentre os possíveis fenômenos associados a esta classe. Duas técnicas foram estudadas de forma a contemplar a detecção, duração e classificação dos fenômenos de VTCDs. Os estudos envolveram a aplicação do cálculo do valor Root Mean Square (RMS) e também da Transformada Wavelet (TW).

Cabe colocar que na literatura consultada, encontram-se muitos trabalhos aplicando tais estratégias na área de QEE. Por exemplo, em Santoso et al. (1996) é apresentada uma abordagem para detectar, localizar e investigar a viabilidade de classificar os diversos tipos de distúrbios de QEE utilizando a TW. A decomposição é realizada usando a técnica de análise multiresolução (AMR), com a Daubechies - 4 (db4) como Wavelet mãe. A escolha desta Wavelet mãe foi devido à mesma ter um suporte mais compacto. O sinal analisado foi decomposto até o quarto nível pela AMR, sendo a detecção e a localização do distúrbio realizada no primeiro nível de detalhe. Os autores também descrevem um possível esquema de classificação dos distúrbios, utilizando o quadrado dos coeficientes da TW, para extrair as características dos sinais distorcidos.

Kopparapu e Chandrasekaran (1998) apresentaram um estudo sobre a aplicação da TWD como ferramenta de detecção e classificação de distúrbios relacionados à QEE. Para os casos testados, foi empregada a decomposição do sinal no primeiro e/ou segundo nível, para a detecção dos mesmos. Para os casos de afundamento, elevação e ruídos, o sinal foi decomposto em outros níveis, para se obter maiores informações do evento. Após a detecção, os autores calcularam o valor RMS e a curva de desvio padrão em cada nível do sinal em análise de modo que estes pudessem ser usados como parâmetros para a classificação dos distúrbios pelos sistemas especialistas.

Xiangxun (2002) sugere uma metodologia dispondo da TW para detectar, quantificar e classificar distúrbios relacionados à QEE. O autor extrai características exclusivas dos distúrbios a partir de informações no domínio do tempo ou da frequência, realizando, posteriormente, uma conversão binária para decimal. Para a classificação, utilizou-se de um vetor característico com cinco elementos, com um código binário, que posteriormente foi convertido para decimal.

Uma comparação entre três formas de caracterização da magnitude de distúrbios de QEE é apresentada em dois trabalhos de Wang et. al. (2002). O cálculo do valor RMS da tensão, o valor de pico da tensão e da componente da tensão fundamental foram introduzidos para quantificar o nível de severidade observado. O valor RMS foi obtido utilizando-se os coeficientes de detalhe da TW. A componente da tensão fundamental foi obtida através da aplicação da transformada rápida de Fourier (TRF). O valor de pico da tensão foi obtido pela comparação do máximo valor absoluto da janela estudada com o maior valor da janela precedente. Os autores concluíram que o cálculo do valor RMS da tensão é apropriado para situações onde harmônicas e/ou flickers (cintilações) são predominantes. Conforme apontado, o valor de pico da tensão pôde ser utilizado como um valor auxiliar para a classificação dos transitórios. Os mesmos afirmaram que a componente fundamental da tensão é a variável apropriada para a caracterização da magnitude da maioria das situações estudadas.

Outro trabalho proposto por Oleskovicz et al (2006) apresenta um estudo comparativo referente à aplicação da TW, transformada de Fourier janelada (TFJ) e redes neurais artificiais (RNAs) em análises associadas à QEE. Os resultados apresentados abrangem a detecção, localização e classificação de fenômenos de elevação, afundamento e interrupção de tensão, transitório oscilatório e ruídos pelas técnicas ci- 
tadas. Cabe ressaltar que foram utilizadas RNAs apenas na etapa de classificação das anormalidades contidas nas situações evidenciadas pelos autores.

No contexto apresentado, vale ressaltar que os trabalhos encontrados na literatura correlata, ilustrados por estes que foram anteriomente mencionados, não contemplam os objetivos almejados nesse trabalho, pois não abordam os fenômenos de QEE de acordo com as legislações brasileiras. Além disso, a utilização do cálculo do valor RMS de um determinado sinal é utilizado apenas para as etapas de classificação. Somando-se à classificação, neste relato, o valor RMS será também empregado para acusar certas descontinuidades nos sinais em análise. Outro ponto importante diz respeito aos estudos comparativos entre as técnicas descritas anteriormente, já que muitas destas foram somente apresentadas como alternativas para o monitoramento de eventos relacionados às VTCDs, sem correlacioná-las às técnicas registradas na literatura.

Contudo, vale destacar que muitas das pesquisas correlacionadas indicam a TW como uma poderosa ferramenta a ser utilizada na análise de sinais elétricos. Desta maneira, o presente trabalho vislumbra a aplicabilidade desta ferramenta frente ao cálculo do valor RMS, visto que este é muito empregado em equipamentos registradores e analisadores de QEE disponíveis comercialmente.

\section{FERRAMENTAS EMPREGADAS NO MONITORAMENTO}

Nesta linha de pesquisa em discussão, foram avaliadas duas técnicas de processamento de sinais, uma dispondo do cálculo do valor RMS, e a outra, da TW.

Os conceitos fundamentais de tais técnicas aplicadas no desenvolvimento deste trabalho, estão registrados nas seções que seguem.

\subsection{Cálculo do valor RMS}

Segundo Johnson et.al. (2000), o valor RMS ou valor eficaz é uma medida estatística da magnitude de uma quantidade variável. A nomenclatura deriva da raiz (Root) quadrada da média (Mean) do valor ao quadrado (Square) da função. O valor RMS pode ser calculado para uma série de valores discretos ou para uma função variável contínua. Neste trabalho será caracterizado o cálculo do valor RMS de um sinal de tensão discreto, sendo o mesmo dado pela Eq. (1), onde $V_{i}$ é a amostra do sinal e $N$ o número de amostras compreendidas no período em análise.

$$
V_{r m s}=\sqrt{\frac{1}{N} \sum_{i=1}^{N} V_{i}^{2}}
$$

\subsection{A Transformada Wavelet Discreta}

A transformada Wavelet é uma poderosa ferramenta de processamento de sinais, que permite analisar um sinal tanto no domínio da frequência como no domínio do tempo (Daubechies, 1990). Do ponto de vista matemático, a transformada Wavelet discreta (TWD) permite decompor, em diversas escalas, um sinal discreto como uma combinação linear de funções ortogonais finitas, chamadas Escala $(\phi)$, e Wavelet $(\psi)$. A função do sinal em representação pode ser como a da Eq. (2) :

$$
f[n]=\sum_{k=0}^{\frac{n}{2 j}-1} R_{j, k}[n] \phi_{j, k}[n]+\sum_{t=1}^{j} \sum_{k=0}^{\frac{n}{2 j}-1} S_{t, k}[n] \psi_{t, k}[n]
$$

Sendo:

$$
\begin{aligned}
& \phi[n]=\sum_{k} h[k] \phi(2 n-k) \\
& \psi[n]=\sum_{k} g[k] \phi(2 n-k)
\end{aligned}
$$

Onde $j$ é o nível de decomposição, $n$ é o $n$-ésimo componente do sinal $f[], k$ é o $k$-ésimo elemento dos filtros, $h[k]$ (filtro passa-baixa) e $g[k]$ (filtro passa-alta) que formam um par de QMF (Quadrature Mirror Filter), sendo $R_{j, k}[n]=\left\langle f, \phi_{j, k}[n]\right\rangle$ e $S_{j, k}[n]=\left\langle f, \psi_{j, k}[n]\right\rangle$ o produto escalar da função $\boldsymbol{f}$ com as funções escala $(\phi[n])$ e wavelet $(\psi[n])$, respectivamente.

Desta maneira, do ponto de vista da engenharia, a TWD nada mais é que um processo de filtragem digital no domínio do tempo (via convolução discreta), seguida por uma redução do número de amostras (downsampling) por um fator 2. Ou seja, basicamente, a TWD decompõe um sinal em bandas de múltiplas frequências sem perder a informação no tempo, provendo desta maneira, uma divisão irregular do plano tempofrequência. A mesma atribui pequenos intervalos no tempo para as componentes de alta-frequência e intervalos maiores, para as componentes de baixa frequência. Esta divisão proporciona uma melhor representação (resolução) do sinal nos domínios da frequência e do tempo.

Ao processo de decomposição em múltiplos níveis, tem-se a técnica de análise multiresolução (AMR). Por meio desta técnica o sinal é decomposto em duas distintas versões. Uma 


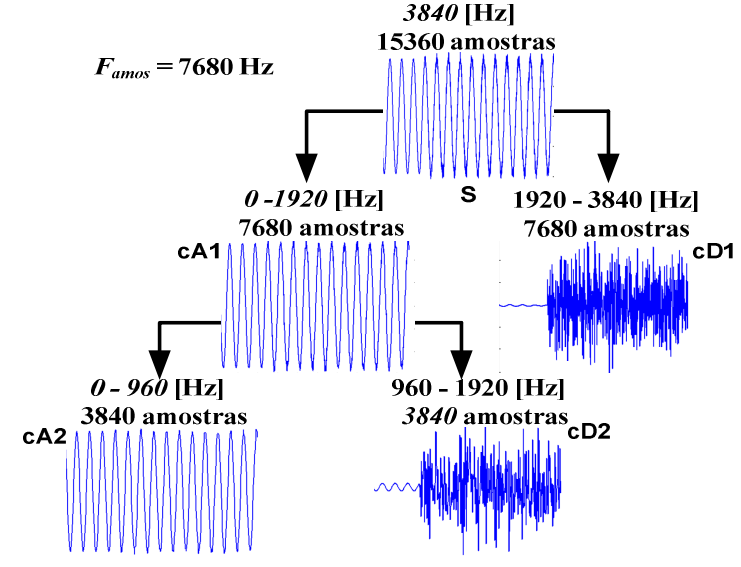

Figura 1: Processo sucessivo de decomposição de um sinal ruidoso, até o segundo nível, mediante a aplicação da AMR.

rica em altas frequências (composto pelos detalhes $-\mathrm{cD}$ ) e outra em baixas frequências (composta pelas aproximações - cA), através de filtros passa alta e passa baixa, respectivamente. Este processo é ilustrado pela Figura 1.

Pela figura, observa-se a decomposição em dois níveis de um sinal de tensão distorcido, caracterizado por uma frequência de amostragem de $7680 \mathrm{~Hz}$ (15.360 amostras, com base na frequência fundamental - $60 \mathrm{~Hz}$ ). Segundo o teorema de $\mathrm{Ny}$ quist (Smith, 1999), o sinal amostrado não deve conter componentes de frequência superior à metade da frequência de amostragem para evitar o fenômeno aliasing (sobreposição de espectros). Por isso, a frequência inicial considerada no sinal em análise está caracterizada por $3840 \mathrm{~Hz}$.

Como pode ser observado pela figura, o primeiro nível de decomposição possui uma melhor representação no tempo e uma baixa resolução na frequência. Isto é devido à representação irregular no domínio tempo-frequência. Com o aumento do nível de decomposição, melhora-se a resolução da frequência e diminui-se a resolução no tempo. Esta redução na resolução é devido à diminuição das amostras disponibilizadas a cada nível em questão.

\section{O SISTEMA DE DISTRIBUIÇÃO REAL SIMULADO E ANALISADO VIA ATP}

Para gerar os casos de VTCDs necessários para a análise do desempenho da abordagem proposta, modelou-se um sistema de distribuição real pertencente a uma concessionária regional, dispondo do software ATP (Alternative Transients Program) (EMTP/ATP, 2002). As grandezas analisadas referemse a valores amostrados das tensões de tal sistema elétrico de distribuição. Deve ser ressaltado que a modelagem deste sistema de distribuição primário, na medida do possível, apre-

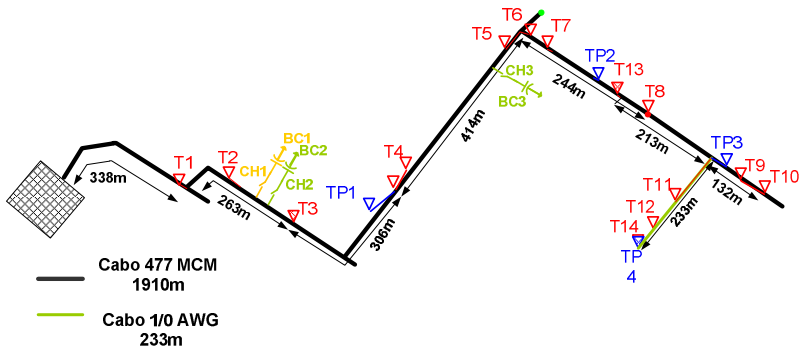

Figura 2: Sistema de distribuição real simulado e em análise.

senta grande semelhança com o encontrado na prática. Inúmeras considerações práticas foram adotadas até a obtenção e teste do mesmo, em um trabalho conjunto entre a concessionária e o grupo de pesquisadores vinculados à universidade. Tal sistema de distribuição é apresentado na Figura 2.

Neste sistema, tem-se caracterizado o tronco principal de um dos alimentadores. Os ramais e a rede secundária foram considerados como cargas equivalentes. Para tal finalidade foram considerados na modelagem computacional 14 transformadores de distribuição (T1-T14), 4 transformadores particulares pertencentes a consumidores da concessionária (TP1TP4) e três bancos de capacitores, sendo um fixo de 1200 kvar, e dois automáticos de 600 kvar cada.

$\mathrm{Na}$ figura, o transformador da subestação (Subestação138/13,8 kV, 25 MVA), os transformadores de distribuição 3 e 13 (T3 e T13, 45 kVA) e o transformador particular 4 (TP 4, $45 \mathrm{kVA}$ ), que aparecem em azul, foram modelados considerando-se suas curvas de saturação. O modelo das cargas do lado secundário destes transformadores segue específicas características que denotam equivalentes das situações reais de carga, segundo as demandas e curvas de cargas típicas repassadas pela própria concessionária. Já os transformadores particulares 1, 2 e 3 (TP1-TP3) foram modelados sem considerar as respectivas curvas de saturação. Logo, as cargas foram referidas ao primário com uma parcela RL em paralelo com um capacitor para a devida correção do fator de potência. Os demais transformadores de distribuição foram modelados somente como cargas RL referidas ao primário, considerando-se um fator de potência geral de 0,95. Cabe ainda destacar que tanto os transformadores de distribuição quanto os particulares apresentam ligações delta-estrela, com resistência de aterramento de zero ohm. O alimentador principal é constituído por cabo nu CA-477 MCM em estrutura aérea convencional, e seus trechos são representados por elementos RL acoplados.

Sobre este sistema, foram geradas situações representativas de operações que caracterizaram interrupções, afundamentos e elevações de tensão. Tais situações permitiram caracterizar os fenômenos desejados conforme as definições apre- 
sentadas pelo PRODIST. Para cada situação caracterizada, considerou-se uma frequência de amostragem de $7,68 \mathrm{kHz}$, o que permite 128 amostras por ciclo dos sinais em análise, tomando como base a frequência fundamental de $60 \mathrm{~Hz}$.

Para gerar um banco de dados representativo, foram aplicadas situações de defeitos do tipo fase-terra sobre o sistema em quatro diferentes pontos. Os pontos em questão referemse a SE (subestação), BC1 (banco de capacitores 1), T13 (transformador de distribuição 13) e TP1 (transformador particular 1), considerando uma variação do ângulo de incidência do defeito $\left(0\right.$ e $\left.90^{\circ}\right)$, da resistência do defeito entre 0,01 e $5 \Omega$ e do tempo de duração do fenômeno $\left(\mathrm{t}_{d}\right)$ entre momentâneo $\left(\mathrm{t}_{d} \leq 3 \mathrm{~s}\right.$ para a interrupção e 1 ciclo $\leq \mathrm{t}_{d} \leq$ 3 s para afundamento e elevação) e temporário $\left(3 \mathrm{~s}<\mathrm{t}_{d} \leq\right.$ 3 min para os três fenômenos associados). As medições foram realizadas na barra secundária da subestação para todas as situações de defeito aplicados. Contudo, além das medições na SE, cabe afirmar que situações de defeito e medições também foram consideradas no ponto de conexão do transformador T13.

Após diversas análises realizadas por meio do software ATP, considerando o sistema de distribuição mencionado, da combinação entre as variáveis apontadas, foi possível selecionar e caracterizar 55 casos de interesse sobre o sistema estudado. Vale comentar que tais situações foram geradas dispondo da interface gráfica do ATPDraw (EMTP/ATP, 2002). Destes 55 casos, realizando uma análise das tensões nas fases "a" e "c", foi possível obter 107 situações de VTCDs para teste das técnicas abordadas neste trabalho. A distribuição dos casos gerados está registrada nas Tabelas 1 e 2.

Destas 107 situações, caracterizaram-se 42 afundamentos (com tensões remanescentes entre 0,1 p.u. e 0,9 p.u.), 52 elevações de tensões, refletidas por valores entre 1,1 p.u. e 1,8 p.u., e 13 de interrupções no fornecimento, caracterizadas por valores abaixo de 0,1 p.u., com durações denotando situações momentâneas e/ou temporárias (Tabela 2).

\section{METODOLOGIAS PROPOSTAS}

Por este reporte, são avaliadas duas metodologias para o monitoramento automático de sinais de tensão relacionados à QEE. Como já colocado, tais metodologias utilizam-se da aplicação do cálculo do valor RMS e da TW, respectivamente. Por estas técnicas, visa-se ao monitoramento de um sistema elétrico a fim de detectar e localizar no tempo, vindo posteriomente a classificar os distúrbios relacionados às VTCDs. Cabe colocar que, para efeito de desenvolvimento e teste, inicialmente a lógica implementada foi compilada em ambiente Matlab (Matlab 7.1, 2005), sendo que, quando da sua validação, pretende-se compô-la em uma linguagem acessível ao setor elétrico.
Tabela 1: Situações de defeito fase-terra aplicadas sobre o sistema de distribuição real em análise.

\begin{tabular}{|c|c|c|c|}
\hline & $\begin{array}{c}\text { Resistência } \\
\text { de defeito } \\
(\text { Ohms })\end{array}$ & $\begin{array}{c}\text { Ângulo de } \\
\text { incidência } \\
\left({ }^{\circ}\right)\end{array}$ & $\begin{array}{c}\boldsymbol{N}^{\text {o }} \text { de casos } \\
\text { gerados }\end{array}$ \\
\hline 1 & 0,01 & 0 e 90 & 8 \\
\hline 2 & 0,03 & 0 e 90 & 5 \\
\hline 3 & 0,07 & 0 e 90 & 6 \\
\hline 4 & 0,08 & 0 e 90 & 2 \\
\hline 5 & 0,09 & 0 e 90 & 1 \\
\hline 6 & 0,1 & 0 e 90 & 5 \\
\hline 7 & 0,3 & 0 e 90 & 6 \\
\hline 8 & 0,7 & 0 e 90 & 4 \\
\hline 9 & 0,9 & 0 e 90 & 1 \\
\hline 10 & 1 & 0 e 90 & 5 \\
\hline 11 & 2 & 0 e 90 & 4 \\
\hline 12 & 3 & 0 e 90 & 5 \\
\hline 13 & 5 & 0 e 90 & 3 \\
\hline & Total & & 55 \\
\hline
\end{tabular}

Tabela 2: Situações representativas de VTCDs geradas para a análise do desempenho da abordagem proposta.

\begin{tabular}{|c|c|c|c|}
\hline $\begin{array}{c}\text { Localização } \\
\text { do defeito }\end{array}$ & Afundamento & Elevação & Interrupção \\
\hline SE & 1 & 9 & 8 \\
\hline CH1 & 10 & 10 & - \\
\hline T13 & 21 & 24 & 5 \\
\hline TP1 & 10 & 9 & - \\
\hline
\end{tabular}

A Figura 3 ilustra o diagrama do modelo computacional desenvolvido dispondo das duas estratégias, sendo este dividido em 4 etapas. As etapas 2 e 3 apresentam rotinas computacionais distintas de acordo com as ferramentas aplicadas (valor RMS e TW). Já para as etapas 1 e 4, as rotinas são comuns e independentes das ferramentas de análise aplicadas.

\subsection{Etapa 1 - Aquisição e condiciona- mento por fase dos dados}

Inicialmente, os sinais de tensão das três fases são registrados e condicionados, ou seja, estes são submetidos a um processo de filtragem segundo o critério de Nyquist (Smith, 1999) e 


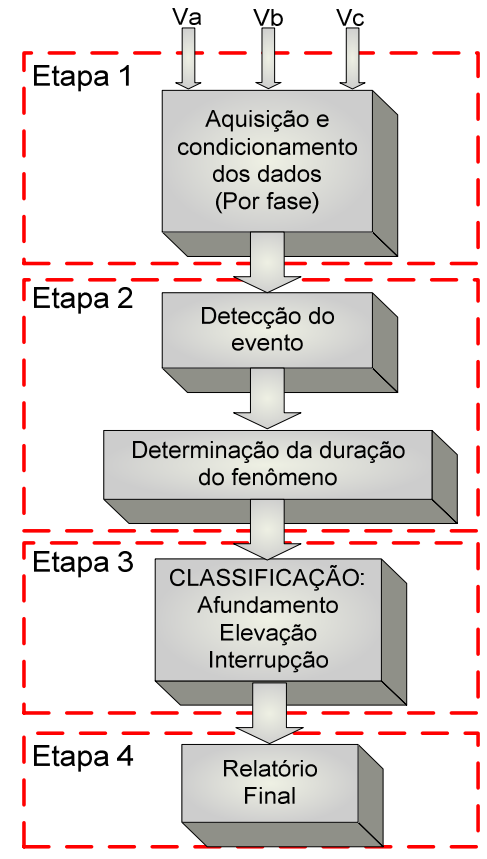

Figura 3: Diagrama do modelo computacional desenvolvido.

posteriormente normalizados para valores entre -1 e 1 p.u., empregando-se como base o valor de pico da tensão em regime permanente. Todo o processo para esta etapa é realizado por meio do "janelamento" do sinal para cada fase. Desta forma caracteriza-se um deslizamento no tempo da janela de dados sobre o sinal em análise. Tal janela deslizante compreende 128 amostras (1 ciclo do sinal), com um passo de movimento de $1 / 4$ de ciclo $(4 \mathrm{~ms})$. Vale ressaltar que todo o processamento a ser realizado entre as etapas 1 e 4 , independente da ferramenta aplicada, deverá ser compatível aos $4 \mathrm{~ms}$ ditados pelo movimento da janela de dados em análise.

\subsection{Etapa 2 - Detecção e estimação da duração do evento}

Para esta etapa, foram desenvolvidas duas estratégias para a detecção e estimação da duração do evento, sendo uma fundamentada no cálculo do valor RMS, e a outra na análise via TW.

Para tal, a janela condicionada na etapa anterior, é apresentada às ferramentas de forma independente, que irão verificar se há alguma descontinuidade sobre o sinal em análise. Quando da acusação de alguma descontinuidade, da maneira como implementado, a lógica (via RMS ou TW) buscará evidenciar o término da mesma.

\subsubsection{Dispondo do valor RMS}

Para a detecção dispondo do valor RMS, calcula-se esse valor para cada janela apresentada, sendo o valor resultante comparado aos limites operativos e aceitáveis para o sistema de distribuição ( 0,9 p.u. a 1,1 p.u.), conforme encontrado no PRODIST. Sendo assim, caso o valor calculado esteja fora dos limites pré-estabelecidos, tem-se a ocorrência de um evento sobre o sistema apontado pela janela em análise. Desta janela, toma-se o instante inicial do evento como sendo o tempo associado a primeira amostra contida na mesma.

Vale colocar que pela lógica implementada, mesmo diante da primeira descontinuidade, a janela continuará em movimento no sentido de apontar a próxima e, caso existam, as demais deformidades no sinal em análise. Considera-se o tempo final de cada evento apontado como aquele relativo à última amostra quando do início de uma nova descontinuidade.

\subsubsection{Dispondo da TW}

Já para a detecção dos eventos dispondo da TW, utilizouse do primeiro nível da decomposição multiresolução com a aplicação da wavelet-mãe Daubechies-4 (db4). Esta possui um suporte mais compacto e uma variação abrupta de amplitude, que permite uma melhor resposta no domínio do tempo.

Para a detecção do início do evento, utiliza-se o valor máximo contido no $1^{\circ}$ nível de detalhe referente à janela analisada. Tal valor é comparado a um limiar pré-estabelecido (Lim_Des_1). Quando da extrapolação deste limiar, o algoritmo indicará que esta janela possui uma anormalidade. O próximo passo é determinar qual é exatamente a amostra que corresponde ao início do evento. Para isso, é realizada uma subtração de dois pares de amostras consecutivas, $x(n+2)-x(n+1)$ e $x(n+1)-x(n)$. Caso a subtração das mesmas seja superior aos limiares estabelecidos (Lim_Des_11) e (Lim_Des_12), determina-se o ponto que representa o início do evento e, em conseqüência, a sua localização no tempo.

Após a detecção do evento (primeira descontinuidade), a TW, via a disponibilização da próxima janela de dados representativa do sinal em análise, procura o próximo valor máximo referente ao $1^{\circ}$ nível de detalhe e o compara a outro limiar (Lim_Desc_2). Da extrapolação de tal limiar, acusa-se uma nova descontinuidade, com procedimento idêntico ao da primeira situação, e, conseqüentemente, o fim deste evento. Com a definição dos tempos de início e fim (primeira e segunda descontinidade), tem-se então a estimação da duração desta ocorrência.

Comenta-se que os limiares empregados via TW, para acusar o início e o fim das descontinuidades, foram definidos por meio de estudos sobre os diversos casos caracterizados e associados à QEE. Os limiares encontrados empiricamente e 
empregados nesta abordagem foram:

- Lim_Desc_1 =0,0035, adotado para indicar a janela com o evento;

- Lim_Desc_11=0,00016 e Lim_Desc_12 = 0,0008, adotados para indicar o início do evento, e

- Lim_Desc_2 = 0,0075, adotado para indicar o fim do evento.

Comenta-se que das características intrínsecas à utilização da TW, verificou-se a possibilidade de indicar precisamente tanto o tempo de início quanto de término da primeira e segunda descontinuidade.

\subsection{Etapa 3 - Classificação dos eventos}

Após a detecção e duração de determinado evento, caso este seja classificado como uma VTCD qualquer, o mesmo passará por uma refinada classificação. Para tal função, foram desenvolvidas duas estratégias fundamentadas no valor RMS e na TW. Para a janela com o evento de VTCD, calcula-se um certo valor dependente da ferramenta associada, que será comparado às faixas apresentadas na Figura 4.

Para a estratégia dispondo do valor RMS, é utilizado o próprio valor RMS da janela calculado na etapa anterior.

Já para a TW, a janela em análise é decomposta até o quinto nível de decomposição. Utiliza-se o quinto nível de aproximação por este conter a frequência de $60 \mathrm{~Hz}$ como valor central, pela qual se caracterizam os fenômenos de VTCDs. Calcula-se então a energia da aproximação desse nível e compara-se este valor à faixa ilustrada na Figura 4 (b).

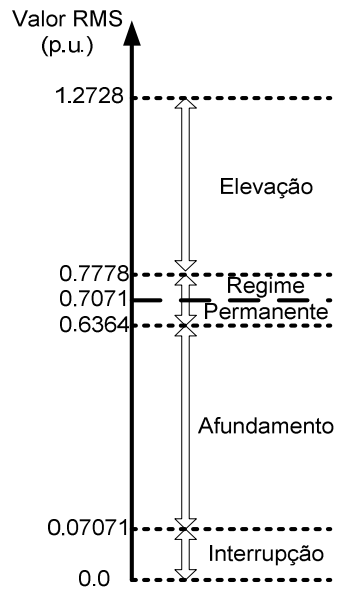

(a) Valor RMS

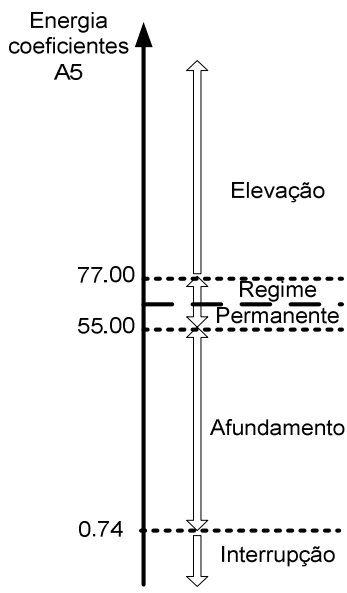

(b) TW
Figura 4: Faixas utilizadas na etapa de classificação.
Ressalta-se que os limites registrados, quando da aplicação da TW na Figura 4, foram obtidos por meio da análise da energia do quinto nível de decomposição dos sinais de tensão. Estes sinais provêem da caracterização de VTCDs geradas sobre o sistema de distribuição em análise, dispondo do software ATP, que estão em acordo com os níveis estabelecidos pelo PRODIST - Módulo 8.

Contudo, pela aplicação da TW e pelo processamento implementado, observa-se que estes limites não são fixos. Os mesmos variam para diferentes frequências de amostragem de aquisição do sinal em análise. Sendo assim, para cada frequência amostral diferente será necessário determinar novos limites. Tal fato ocorre porque o número de amostras, o nível de decomposição que contém a frequência de $60 \mathrm{~Hz}$ como valor central, e a amplitude no nível de aproximação se modificam de acordo com o processo de aplicação da TW (Figura 1).

Sendo assim, uma estratégia para se determinar tais limites deve contemplar as seguintes etapas:

- Gerar computacionalmente e/ou registrar situações de VTCDs de interesse, com o valor da frequência amostral desejada, e com a amplitude do sinal de tensão caracterizando os limites dos afundamentos (superior ou igual a 0,1 p.u. e inferior a 0,9 p.u.), das elevações (superior a 1,1 p.u.) e das interrupções de tensões (inferior a 0,1 p.u.), conforme o apresentado pelo PRODIST.

- Pela aplicação da TW, determinar o nível de decomposição da aproximação que contenha a frequência de 60 $\mathrm{Hz}$ como valor central.

- Calcular a energia da aproximação desse nível para os casos citados. Os valores destas energias deverão indicar os valores limites para a classificação dos fenômenos, como por exemplo, os apresentados na Figura 4.

\subsection{Etapa 4 - Relatório final}

Para o período de monitoramento analisado, ambas as estratégias estudadas retornam um relatório contendo uma descrição sumarizada de todos os eventos ocorridos. Dentre os apontamentos tem-se a identificação temporal (início e fim das descontinuidades) e a classificação dos fenômenos associados à QEE, focados em VTCDs.

Vale lembrar que ambas as metodologias (RMS e/ou TW, Etapas de 1 a 4) podem ser aplicadas de forma independente às três fases do sistema em análise, retornando, como resposta, todas as variáveis indicadas. 


\section{RESULTADOS}

Conforme anteriormente comentado, frente às metodologias estudadas para o monitoramento automático dos sinais elétricos, foram testadas diversas e prováveis situações sobre o sistema de distribuição real (Figura 2) geradas, dispondo do software ATP, via a interface gráfica do software ATPDraw.

\subsection{Etapa 2 - Detecção e estimação da duração do evento}

A Figura 5 ilustra um caso em que os sinais de tensão registrados na subestação referem-se a uma situação de defeito envolvendo a fase "a" com conexão ao terra, aplicada no ponto primário de conexão do transformador de distribuição T13, com resistência de defeito de $0,01 \Omega$ e ângulo de incidência de $90^{\circ}$. Esta situação possui como valores para os instantes inicial e final das descontinuidades 0,5013 e 0,9079 segundos, respectivamente.

Mediante tal situação aplicada, observa-se um afundamento de tensão na fase "a" e, em uma das fases sãs do sistema, neste caso a fase "c", uma elevação de tensão (Figura 5).

Analisando-se apenas a fase "a", onde se caracterizou o afundamento da tensão (Figura 6), é possível visualizar a janela apontada pelos algoritmos dispondo do valor RMS e da TW, indicando os pontos de início e fim do distúrbio.

A janela de dados contendo o início do evento foi apontada pelo algoritmo usando o valor RMS em 0,4916 segundos, sendo o final do mesmo indicado pela janela a partir de 0,9041 segundos.

Já a TW aponta a janela de início no instante de tempo igual a 0,4833 segundos e o fim a 0,9041 segundos.

Vale comentar que, inicialmente, as duas metodologias detectam as janelas que contêm as descontinuidades no sinal de tensão e não os instantes exatos de início e fim das mesmas. Contudo, verificou-se que a TW detectou a janela em que se inicia o distúrbio aproximadamente $1 / 2$ ciclo antes do

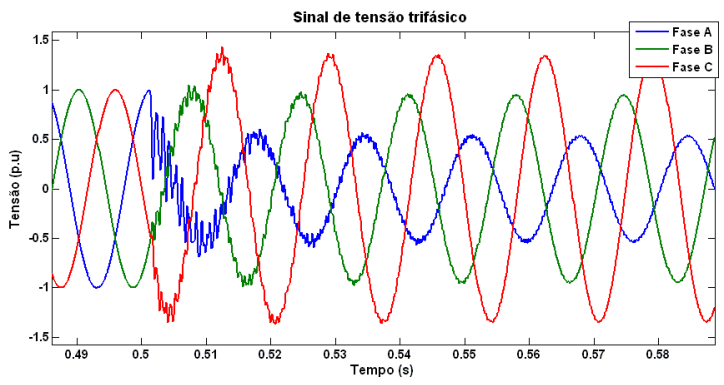

Figura 5: Sinal de tensão trifásico.
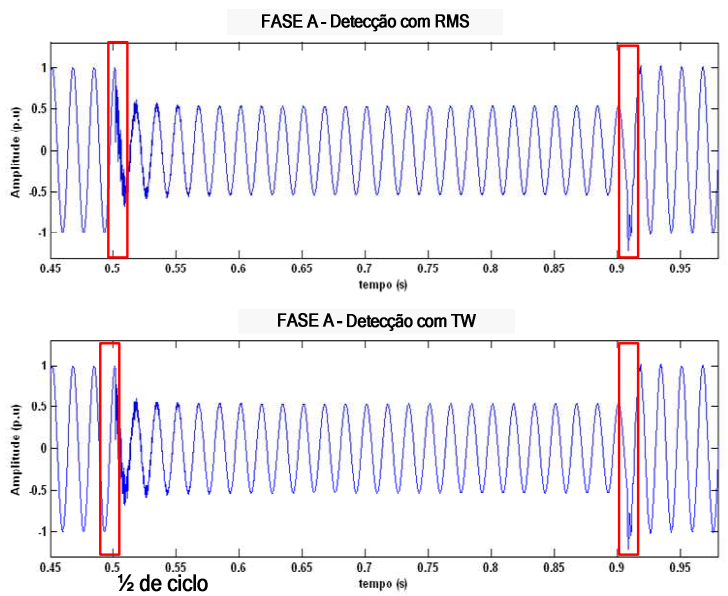

Figura 6: Detecção dos instantes iniciais e finais do afundamento de tensão caracterizado, dispondo do valor RMS e da TW.

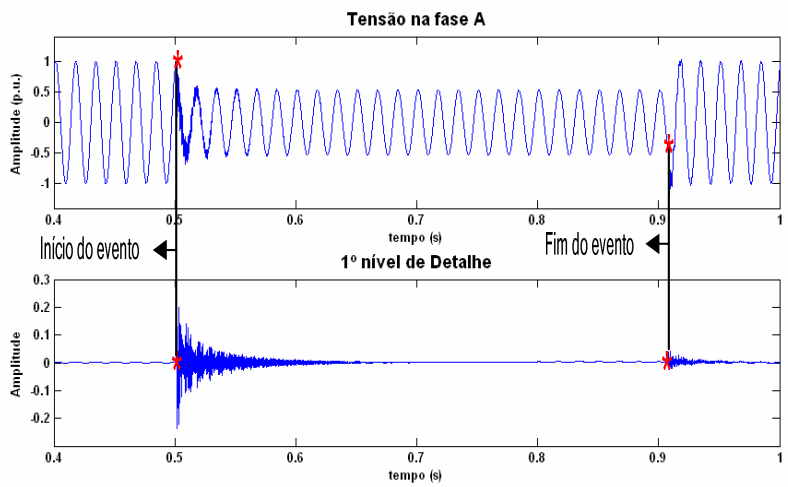

Figura 7: Detecção dos instantes de descontinuidades gerados pelo afundamento de tensão e capturados pela aplicação da TW.

algoritmo usando o valor RMS.

Com relação à identificação da janela em que há o fim do evento de VTCD, da maneira como a lógica foi implementada e já comentada, ambos os algoritmos apresentaram a mesma resposta.

Afirma-se que para todos os 107 casos testados (Tabela 2), obteve-se o mesmo comportamento apresentado.

Com respeito aos instantes exatos da primeira (instante inicial) e segunda (instante final) descontinuidades indicados pela TW para a situação ilustrada na Figura 6, os mesmos são agora ilustrados pela Figura 7.

Pela aplicação da lógica comentada, os tempos estimados pela TW foram: 
- primeira descontinuidade (início do evento): 0,5014 segundos e

- segunda descontinuidade (fim do evento): 0,9079 segundos.

Para o conjunto das situações avaliadas, o valor RMS veio a apresentar um erro médio para a detecção do ínicio do evento de $1,0542 \%$ e a TW de $0,1533 \%$. Já com relação ao fim do evento, o algoritmo fundamentado no valor RMS e na TW veio a apresentar um erro médio de $0,987 \%$ e $0 \%$, respectivamente.

Afirma-se que todas as janelas indicativas da primeira e/ou segunda descontinuidade foram corretamente apontadas pelas duas ferramentas frente aos 107 casos avaliados. Contudo, evidencia-se que para aplicações nas quais não há necessidade de se conhecer o exato instante de tempo em que se inicia o evento, o valor RMS se mostra mais adequado pela sua simplicidade e rapidez no processamento. Porém em situações em que se faz necessário conhecer a duração do evento, a TW se mostra muito eficiente.

Sendo assim, por meio da comparação dos resultados obtidos entre as duas ferramentas, observou-se a eficácia da TW em detrimento do valor RMS que dependerá dos propósitos almejados.

\subsection{Etapa 3 - Classificação dos eventos}

Para a etapa de classificação também foram testadas as estratégias fundamentadas no valor RMS e na TW.

Coloca-se que, após a detecção da primeira descontinuidade sobre o sinal, a classificação dos eventos é realizada a cada nova janela móvel do sinal amostrado.

Para se dispor das duas técnicas, calcula-se o valor RMS e o valor da energia da aproximação do quinto nível de decomposição pela TW. Verifica-se então em que faixa está esse valor segundo a Figura 4. Destes valores, obtém-se a classificação do tipo de distúrbio ocorrido sobre o sinal elétrico analisado. As Figuras 8, 9 e 10 exemplificam esse processo para uma situação de afundamento, elevação e de interrupção da tensão, respectivamente.

A Figura 8 ilustra um afundamento de tensão na fase "a" provocado por um defeito envolvendo a fase "a" com conexão ao terra no transformador T13, com a medição tomada na subestação.

A Figura 9 ilustra uma situação de elevação de tensão na fase "c" devido ao afundamento ocorrido e ilustrado pela figura anterior.
A interrupção ilustrada pela Figura 10 foi provocada pelo mesmo defeito referenciado nas Figuras 8 e 9, porém, com a medição tomada no ponto de defeito. A resistência de defeito considerada nessa situação foi de $0,01 \Omega$.

Vale destacar das situações estudadas que há uma incerteza para se detectar e classificar a janela em que se inicia o evento de VTCD pela técnica do valor RMS. Isso ocorre porque essa técnica faz um média estatística da magnitude de uma quantidade variável, que neste caso é um sinal elétrico senoidal com distúrbio. Portanto, só se evidencia um evento, por meio dessa técnica, na janela que contiver a maioria das amostras com o distúrbio caracterizado. Por outro lado, a TW apresenta mais precisão na classificação já que ela permite identificar, além da janela, a amostra que evidencia o início da descontinuidade.

Na Tabela 3 estão registrados os resultados referentes aos 107 casos avaliados que dizem respeito à etapa de classificação. Esses resultados foram extraídos dos relatórios gerados pelo algoritmo quando da execução da Etapa 4. Com relação a todos os relatórios obtidos pela execução do algoritmo para monitorar as 107 situações avaliadas, observou-se uma grande eficiência das duas ferramentas estudadas. A classificação do evento incidente no sinal de tensão também pode ser conferida de forma visual pela Figuras 8, 9 e 10, ou por meio dos resultados apresentados pelos sistemas propostos em forma de relatório.

Tabela 3: Classificação dos eventos de VTCDs segundo o valor RMS e da TW.

\begin{tabular}{|c|c|c|c|}
\hline \multirow{2}{*}{ Ferramenta } & \multicolumn{3}{|c|}{ Acertos (\%) } \\
\cline { 2 - 4 } & Afundamento & Elevação & Interrupção \\
\hline Valor RMS & 100 & 100 & 100 \\
\hline TW & 100 & 100 & 100 \\
\hline
\end{tabular}

\section{CONCLUSÃO}

Da maneira como foi conduzida, esta pesquisa evidenciou certas particularidades no cálculo do valor RMS e da TW, quando aplicadas ao monitoramento automático de fenômenos associados às VTCDs.

Vale destacar no contexto apresentado, a eficiência na detecção e localização das VTCDs por meio do emprego da TW, já que a mesma propicia um certo refinamento em apontar o tempo de ocorrência das "n" descontinuidades que podem estar presentes em um dado sinal. Tal comentário aponta a habilidade e potencialidade da técnica para aplicações no monitoramento de sinais elétricos em tempo real.

Como apresentado, salienta-se que a TW só necessita do pri- 

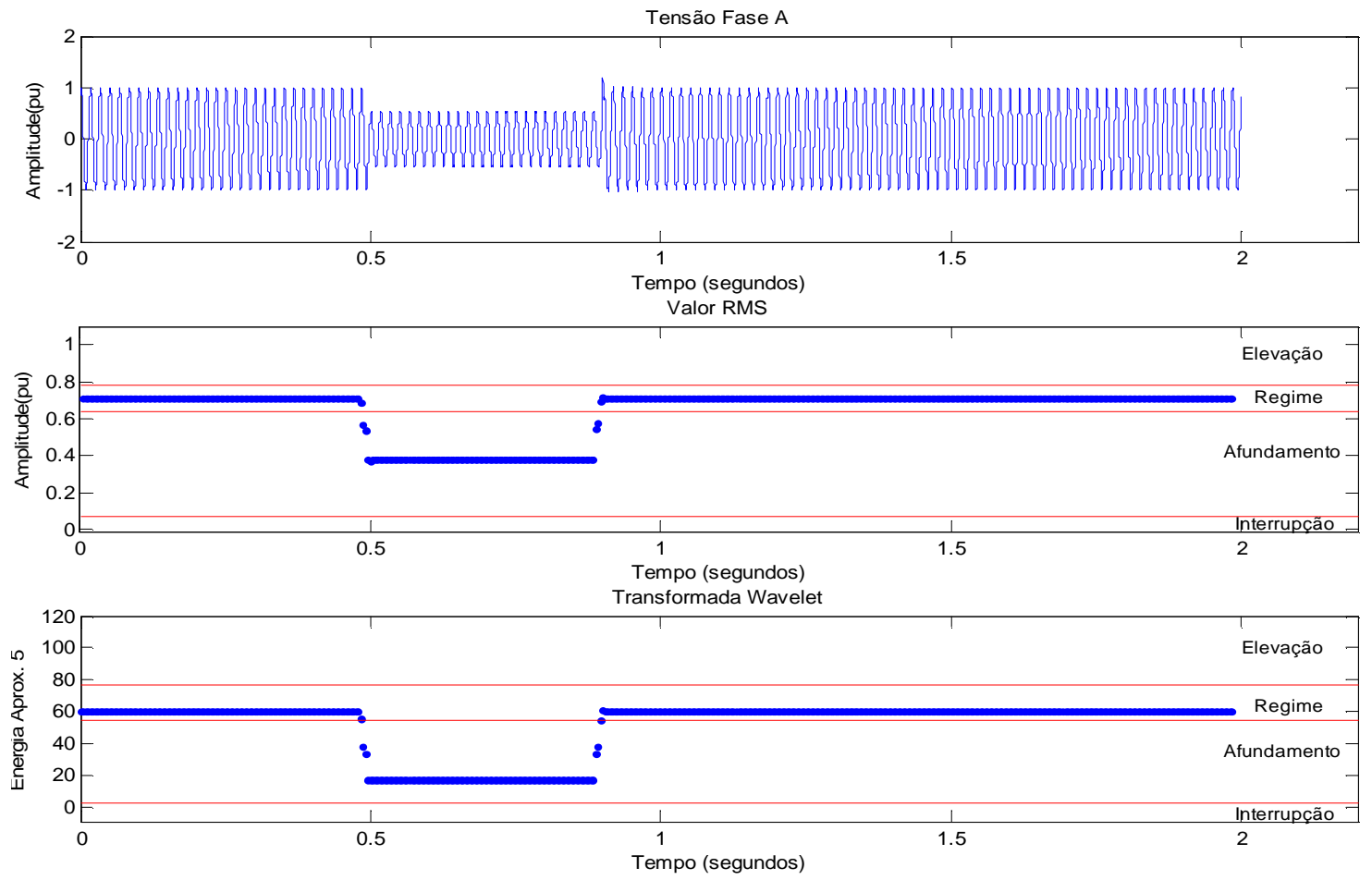

Figura 8: Análise de uma situação de afundamento de tensão (valor remanescente de 0,53 p.u.) evidenciada sobre o sistema de distribuição real em análise.
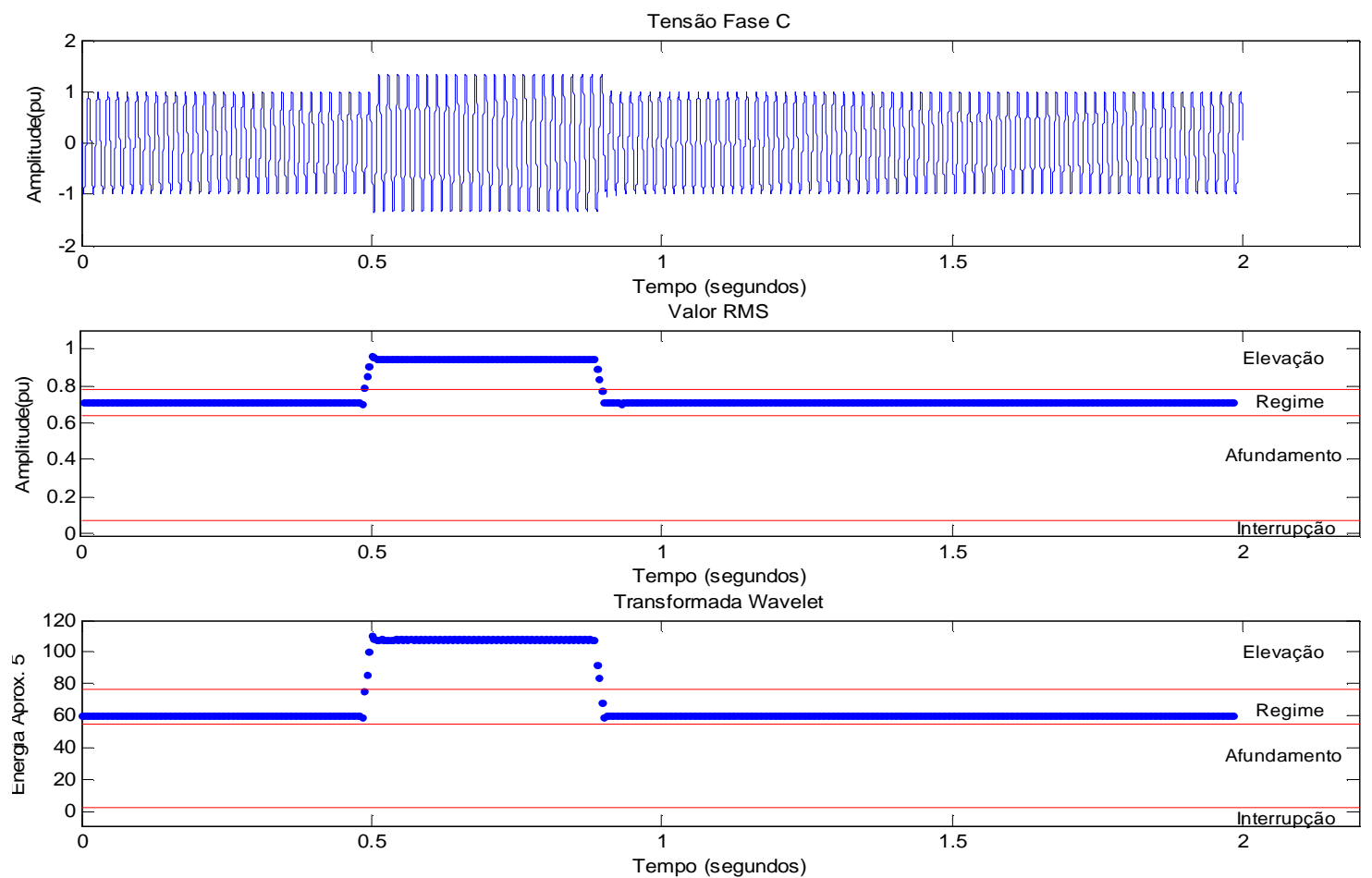

Figura 9: Análise de uma situação de elevação de tensão de 1,34 p.u. evidenciada sobre o sistema de distribuição real em análise. 

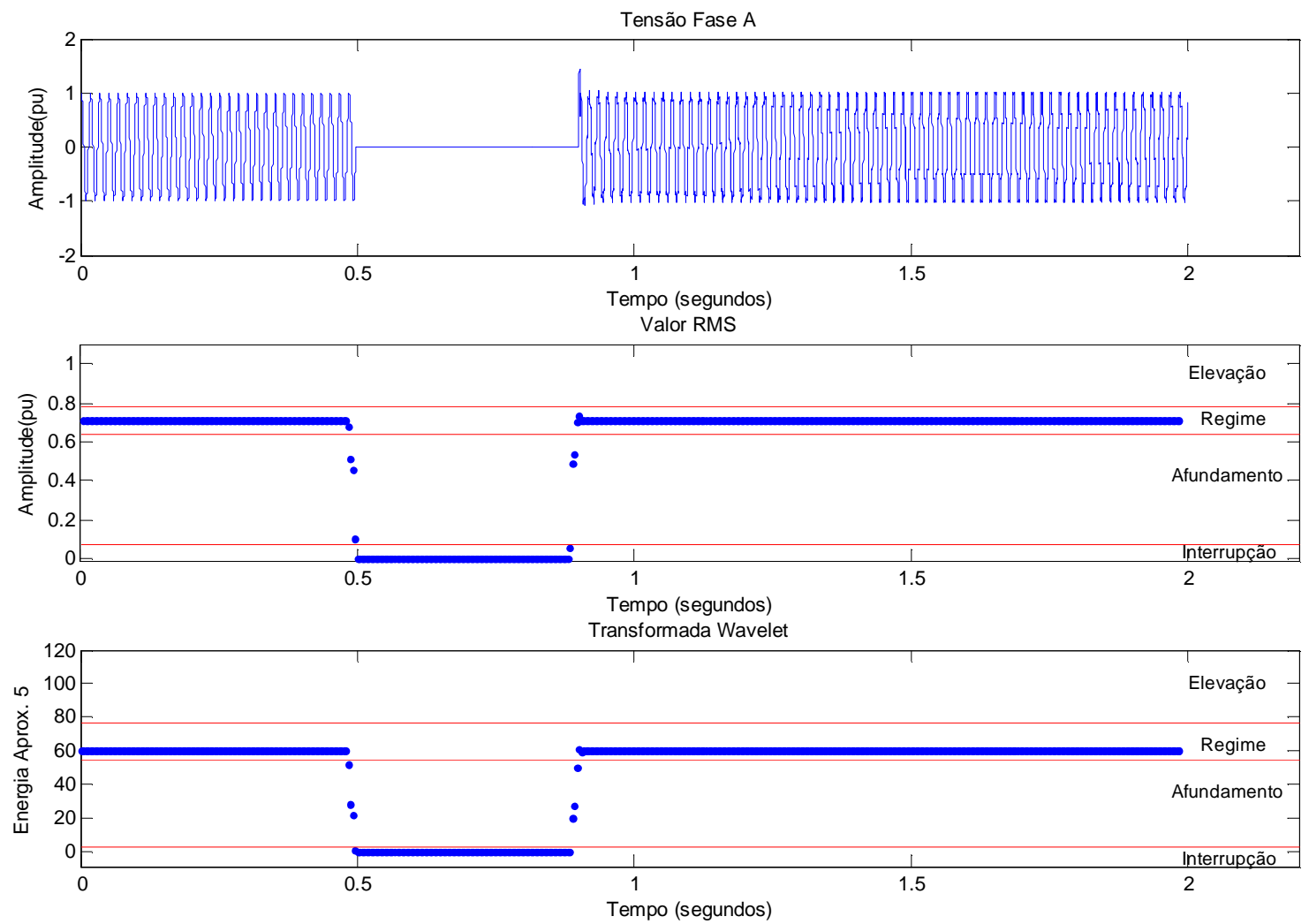

Figura 10: Análise de uma situação de interrupção de tensão evidenciada sobre o sistema de distribuição real em análise.

meiro nível de decomposição do sinal para a etapa de detecção e é possível, por este, estimar o tempo da primeira (início do fenômeno), segunda (e se for o caso, do final do evento) e outras descontinuidades sobre o sinal.

Por outro lado, o algoritmo fundamentado no valor RMS, por si só, não viabiliza o refinamento desejado para tais apontamentos.

Já para a etapa de classificação dos eventos, o valor RMS apresentou simplicidade quando comparado à TW para discriminar entre os possíveis fenômenos associados às VTCDs. Isso pode ser colocado pelo fato da TW necessitar da decomposição até o quinto nível para se obter a classificação correta do(s) evento(s) presente(s) no sinal. Contudo, fica claro que tal fato não inviabiliza de maneira alguma a sua aplicação, já que, pelo processo de decomposição, filtram-se os sinais com frequência superiores a da fundamental, permitindo uma melhor caracterização das VTCDs.

É interessante relembrar que o uso do valor RMS é muito difundido pelos fabricantes de equipamentos para a análise e monitoramento da QEE devido à simplicidade e eficiência, principalmente na etapa de classificação dos fenômenos associados.
Contudo, destaca-se que o emprego da TW apresenta potencialidades quando se necessita de um maior número de detalhes para o monitoramento das VTCDs, permitindo a correlação no plano tempo-frequência, abrindo espaço para a implementação de outras abordagens, diferentes das que aqui foram reportadas. Como, por exemplo, quando do apontamento pela TW das descontinuidades observadas nos sinais, fica evidente a possibilidade de se trabalhar com um tamanho da janela de dados flexível. Sendo assim, este tamanho da janela, poderá ser adaptado para melhor representar os fenômenos, sejam estes únicos ou não nos sinais em observação. Como consequiência, do tamanho da janela especificado para caracterizar determinado fenômeno, poderá também ser indicado qual ferramenta será propícia à análise desejada, visto que, muitas das usualmente empregadas apresentam dificuldades quanto se reduz e/ou se aumenta a janela de dados considerada.

Sendo assim, para a escolha dentre as duas ferramentas apresentadas, já que ambas apresentaram um ótimo desempenho, tem-se somente que pensar no seguinte questionamento: Quanto se deseja explorar um determinado sinal? Para uma resposta baseada em uma abordagem não tanto rica em detalhes, pode-se apontar o valor RMS. Em contrário, buscandose uma maior riqueza, tamanho flexível da janela de dados, 
uma melhor resolução no tempo e/ou na frequência, que venham a possibilitar estudos futuros, pode-se apontar a TW como candidata.

\section{AGRADECIMENTOS}

Os autores agradecem o apoio financeiro recebido por parte do CNPq e da CAPES em fases distintas de desenvolvimento do trabalho. Também agradecem ao Departamento de Engenharia Elétrica da Universidade de São Paulo - Campus de São Carlos, por toda a infra-estrutura necessária para a elaboração deste trabalho.

\section{REFERÊNCIAS}

ANEEL (2000) - Resolução ANEEL n ${ }^{o}$ 024/2000 - Continuidade da distribuição de energia elétrica. Disponível em: http://www.aneel.gov.br/biblioteca/ pesquisadigit.cfm. Acessado em 20 mar. 2008.

ANEEL (2001) - Resolução ANEEL n ${ }^{o}$ 505/2001 Conformidade dos níveis de tensão. Disponível em: http://www.aneel.gov.br/biblioteca/pesquisadigit.cfm. Acessado em 20 mar. 2008.

ANEEL (2009) - Procedimentos de Distribuição de Energia Elétrica no Sistema Elétrico Nacional - PRODIST. Disponível em: http://www.aneel.gov.br/82.htm. Acessado em 26 fev. 2009.

Comitê Argentino de Usuários de EMTP/ATP (CAUE) (2002). Alternative Transients Program Rule Book.

Daubechies, I. (1990); "The Wavelet Transform, TimeFrequency Localization and Signal Analysis", IEEE Transaction on Information Theory, v. 36, n. 5, pp. 9611005.

Johnson, D. E.; Hilburn, J. L.; Johnson, J. R. (2000); Fundamentos de Análise de Circuitos Elétricos. Editora LTC, 4a edição, pp. 304-305.

Oleskovicz, M., Coury, D. V., Carneiro, A. A. F. M., Souza, S. A., Filho, O. D., Arruda. E. F. (2006). Estudo Comparativo de Ferramentas Modernas de Análise aplicadas à Qualidade da Energia Elétrica. Revista Controle \& Automação, v. 17, n. 3, pp. 331-341.

Matlab - The language of Technical Computing. Matlab 7.1.0.246 (R14) Service Pack 3, August 02, 2005.

Santoso, S., Powers, E. J., Grady, W. M., Hofmann, P. (1996). Power Quality Assessment Via Wavelet Transform Analysis. IEEE Transaction on Power Delivery, v. 11, n. 2, pp. 924-930.
Kopparapu, C.; Chandrasekaran, A. (1998). A study on the application of wavelet analysis to power quality. In: Proceedings of the Thirtieth Southeastern Symposium on System Theory, pp.350 - 353.

Smith, S. W.; The Scientist and Engineer's Guide to Digital Signal Processing. California Technical Publishing, n.2, pp. 39-44.

Xiangxun, C. (2002). Wavelet-based detection, localization, quantification and classification of short duration power quality disturbances, IEEE Power Engineering Society Winter Meeting, 2002. v. 2, pp. 931-936.

Wang, Z.Q., Zhou, S.Z., Guo, Y.J. (2002). Comparisons on ways of magnitude characterization of power quality disturbances, In: Lescope 02 - Large Engineering Systems Conference on Power Engineering, pp. 178-183.

Wang, Z.Q., Zhou, S.Z. (2002). Comparative study on power quality disturbance magnitude characterization, In: Powercon 2002 - International Conference on Power System Technology, v. 1, pp. 106-111, 13-17. 